\title{
An activated Notch1 signaling pathway inhibits cell proliferation and induces apoptosis in human esophageal squamous cell carcinoma cell line EC9706
}

\author{
ZHAOMING LU, HONGTAO LIU, LEXUN XUE, PEIRONG XU, TIANXIAO GONG and GUIQIN HOU \\ Laboratory for Cell Biology, The First Affiliated Hospital, Zhengzhou University, Henan 450052, P.R. China
}

Received September 24, 2007; Accepted November 5, 2007

\begin{abstract}
Previous studies have demonstrated that Notch1 signaling pathway plays a major role in maintaining the balance of cell proliferation, differentiation and apoptosis, and is closely associated with tumorigenesis. However, roles of Notch1 signaling pathway in esophageal squamous cell carcinoma (ESCC), which is a common cause of mortality in China, remain poorly understood. Therefore, a novel strategy for seeking a rational molecular therapeutic target for ESCC is urgently needed. The purpose of this study is to examine the effect of the active Notch1 signaling pathway on the proliferation and apoptosis of ESCC cells and to investigate the underlying molecular mechanisms in carcinogenesis of the esophagus. The results revealed that a constitutively activated Notch1 signaling pathway was observed in ESCC cell line EC9706, through a pcNICD vector mediated expression system. Clearly, the activated Notch1 signaling pathway gave rise to proliferation suppression of the cells, accompanied with a cell cycle inhibition at the $\mathrm{G}_{0} / \mathrm{G}_{1}$ phase and apoptosis. In contrast to the expression of CDK2, cyclin D1 and cyclin E observed in EC9706 cells untreated and transfected with pcDNA3.1, there was a markedly decrease in the cells stably expressing Notch 1 NICD. Up- and down-regulations of GSK $3 \beta$ and $ß$-catenin, respectively, indicated that Notch1 inhibited proliferation and induced apoptosis of EC9706 cells through Wnt-mediated signaling pathway. These findings suggest that Notch1 signaling pathway may participate in carcinogenesis of the esophagus.
\end{abstract}

\section{Introduction}

Esophageal squamous cell carcinoma (ESCC) is one of the most frequently diagnosed cancers in developing countries, especially in China (1). However, the understanding of

Correspondence to: Professor Lexun Xue, Laboratory for Cell Biology, The First Affiliated Hospital, Zhengzhou University, 40 Daxue Road, Zhengzhou, Henan 450052, P.R. China

E-mail: xuelx@371.net

Key words: esophageal squamous cell carcinoma, Notch1 signaling pathway, cell cycle, cell apoptosis etiology and mode of carcinogenesis of this disease is still lacking (2). Although therapy strategies have been improved, the prognosis of patients with ESCC is still poor. Moreover, cells of ESCC are known to develop resistance to chemotherapeutic drugs, thus resulting in a dramatic decrease in the 5-year survival rate for ESCC (3). Obviously, a better understanding of the molecular mechanisms in carcinogenesis and progression of ESCC helps to improve the prognosis of patients with ESCC.

Notch protein is a transmembrane receptor family that is structurally and functionally conserved from worms to human. Notch1 receptor was first identified in Drosophila as a gene involved in neuronal cell fate decision, and currently it is known to regulate the fate decisions of developing cells in various tissues during embryogenesis as well as in postnatal stages (4). Upon binding to its ligand, Notch1 protein is proteolytically cleaved, releasing a Notch1 intra-cellular domain (NICD), which then translocates into the nucleus (5-7). The NICD associates with transcriptional factors known as $\mathrm{Su}(\mathrm{H}) / \mathrm{CBF} 1$, regulating the expression of target genes and successively modulating the development of cells $(8,9)$.

Previous studies have revealed that the Notch1 signaling pathway, as one of key signaling pathway regulating cell differentiation, played a key role in cell proliferation, differentiation and apoptosis that affect the development and function of many organs $(10,11)$. At present, insights into links between Notch1 signaling pathway and cancer are rapidly expanding. The Notch1 signaling pathway has been associated with several human cancers, including cervical cancer, lung carcinoma and neuroblastoma $(12,13)$. Also, aberrant Notch signaling pathway plays a role in other types of leukemia (14) and mucoepidermoid tumors $(15,16)$. In addition, Notch1 can be a dominant oncogene as well as a tumor suppressor gene, depending on the cellular context (17). The rapidly increasing knowledge about Notch1 signaling pathway in cancer will hopefully provide a basis to explore the possibility of using Notch signaling as a therapeutic target in cancer (13).

Since the Notch1 signaling pathway in ECSS has not been investigated, it prompted the authors to investigate the role of Notch1 signaling pathway in carcinogenesis of the esophagus. The purpose of this study was to establish whether a constitutively activated Notch1 signaling pathway exists in ECSS, and to investigate the effects of Notch1 on growth and apoptosis of the EC9706 cells. 


\section{Materials and methods}

Cell line and cell culture. A poorly differentiated ESCC cell line EC9706 was provided by the State Key Laboratory of Molecular Oncology, Chinese Academy of Medical Sciences (Beijing, P.R. China). The cells were cultured in RPMI-1640 medium (Bibco-BR2, Rockville, USA) supplemented with $10 \%$ fetal bovine serum (FBS) (HyClone Laboratories, Logan, USA), $100 \mathrm{U} / \mathrm{ml}$ penicillin and $100 \mu \mathrm{g} / \mathrm{ml}$ streptomycin at $37^{\circ} \mathrm{C}$ in the presence of $5 \% \mathrm{CO}_{2}$, as described in the previous studies from our laboratory $(18,19)$.

Immunocytochemical analysis. The immunocytochemical analysis was performed using the SP kit (Santa Cruz, USA) according to the manufacturer's instructions. Briefly, EC9706 cells were plated on several sterile glass slides and incubated at $37^{\circ} \mathrm{C}$ for $24 \mathrm{~h}$ in presence of $5 \% \mathrm{CO}_{2}$. The slides were rinsed three times in phosphate-buffered saline (PBS, pH 7.4) and fixed with $4 \%$ formaldehyde at room temperature (RT) for $10 \mathrm{~min}$. After rinsing in PBS and treatment with $3 \% \mathrm{H}_{2} \mathrm{O}_{2}$ for $10 \mathrm{~min}$, the cells were blocked with $5 \%$ normal goat serum for $30 \mathrm{~min}$ in a humidified box at RT to eliminate nonspecific binding, and then incubated with Notch1 antibody (1:100) (Santa Cruz), and PBS as a control, respectively, at $4^{\circ} \mathrm{C}$ overnight. After the slides were rinsed three times in PBS and incubated with corresponding secondary antibody for $30 \mathrm{~min}$, they were developed with a $0.03 \%$ DAB solution. Subsequently, photomicrographs (magnification, x400) were taken immediately.

Construction of expression vector and transfection. Human Notch1 (NICD) gene (corresponding to amino acids 17592444 of GenBank NM_017617) was amplified by PCR using Notch1-specific primers, 5'-CGCGGATCCATGCGCAAG CGCCGGCGGCAGCAT-3' and 5'-ACGTCTAGACACG TCTGCCTGGCTCGG-3' (underlined showing BamHI and $X b a \mathrm{I}$, respectively), using human placenta tissue cDNA as a template, and PCR was performed using LA Taq ${ }^{\mathrm{TM}}$ System (Takara, P.R. China) in 30 cycles of $94^{\circ} \mathrm{C}$ for $50 \mathrm{sec}, 58^{\circ} \mathrm{C}$ for $50 \mathrm{sec}$ and $72^{\circ} \mathrm{C}$ for $90 \mathrm{sec}$. The PCR product (about $2.0 \mathrm{~kb}$ ) was inserted into the $\mathrm{Bam} \mathrm{HI} / \mathrm{XbaI}$ restriction sites of the mammalian expression vector pcDNA3.1 (+) (Invitrogen, USA), resulting in a vector pcDNA3.1/Notch1, named pcNICD. Meanwhile, the construct was sequenced in both directions to verify the correctness of the vector.

To obtain stable transfectants, EC9706 cells seeded at a density of $2 \times 10^{6}$ cells $/ \mathrm{ml}$ into a 6 -well plate $(4 \mathrm{ml} / \mathrm{well})$ were cultured for $24 \mathrm{~h}$, and then transfected with $2.5 \mu \mathrm{g}$ of the pcNICD and $5 \mu \mathrm{l}$ of Lipofectamine 2000 (Invitrogen) according to the manufacturer's instructions. After transfection, cells were selected for 2-5 days with G418 at $400 \mu \mathrm{g} / \mathrm{ml}$. As soon as mortality rate of the cells untreated was $100 \%$, selection was continued with $\mathrm{G} 418(200 \mu \mathrm{g} / \mathrm{ml})$ for 2-3 more weeks until individual colonies appeared.

Immunofluorescence. Permeabilization and fixation of cultured cells were carried out with Triton X-100 and paraformaldehyde, respectively, as described previously (20). After a rehydration with multiple rinses in PBS, the cells stably expressing NICD were treated with PBS containing
$4 \%$ bovine serum albumin, immunofluorescence staining of cells was carried out with rabbit anti-Notch1 polyclonal antibody (Santa Cruz) (1:100) as the primary antibody and FITC-conjugated goat anti-rabbit immunoglobulin $\mathrm{G}(\mathrm{H}+\mathrm{L})$ antibody as the secondary antibody, respectively. Immunostained cells were washed thoroughly with PBS and mounted in a buffer containing $0.1 \mathrm{M} \mathrm{PBS}, \mathrm{pH} 8.0,2 \%$ n-propyl gallate and $60 \%$ glycerol. Photomicrographs (magnification, $\mathrm{x} 400$ ) were taken using a Nikon microscope equipped with epifluorescence.

Semi-quantitative $R T$-PCR. Total RNA was isolated from untreated and transfected EC9706 cells by TRIzol reagent (Invitrogen) according to the manufacturer's instructions, and then subjected to first-strand cDNA synthesis with AMV first strand DNA synthesis kit (Biotech Co., Shanghai, P.R. China). Briefly, $1 \mu \mathrm{g}$ of the isolated RNA was reversely transcribed to cDNA at $42^{\circ} \mathrm{C}$ for $1 \mathrm{~h}$ in $20 \mu \mathrm{l}$ of reaction mixture with $1 \mu 1 \mathrm{AMV}$ reverse transcriptase, $1 \mu 1$ random hexamer, $4 \mu 15 \mathrm{X}$ reaction buffer, $2 \mu 1 \mathrm{dNTP}(10 \mathrm{mM})$ and $1 \mu 1$ Rnase inhibitor $(20 \mathrm{U} / \mu 1)$. The PCR amplification mixture $(25 \mu \mathrm{l})$ consisted of $1 \mu \mathrm{l}$ cDNA mixture, $0.5 \mathrm{U}$ LA Taq DNA polymerase, $2.5 \mu 1$ 10X LA PCR buffer, $2.5 \mathrm{mM}$ dNTP mixture and $50 \mathrm{pM}$ sense and antisense primers each (Table I). Actin was used as an internal control. PCR was carried out as follows: initial denaturation at $94^{\circ} \mathrm{C}$ for $2 \mathrm{~min}$, followed by $30 \mathrm{sec}$ at $94^{\circ} \mathrm{C}, 30 \mathrm{sec}$ at each anneal temperature for different genes (Table I), and $50 \mathrm{sec}$ at $72^{\circ} \mathrm{C}$ for a total of 30 cycles and a terminal extension at $72^{\circ} \mathrm{C}$ for 6 min. After amplification, $10 \mu 1$ of PCR products were resolved on a $1 \%$ agarose gel. DNA bands were visualized by UV light and documented with a Gene Tools (Model P67UA).

Cell proliferation assay by cell counting kit-8 (CCK-8). CCK-8 is a sensitive non-radioactive colorimetric assay for determining the number of viable cells in cell proliferation and cytotoxicity assays. CCK- 8 solution is added directly to the cells; no pre-mixing of components is required. The detection sensitivity of cell proliferation assays using CCK-8 is higher than assays using the other tetrazolium salts such as MTT, XTT, MTS or WST- 1 . Since the CCK- 8 solution is very stable and it has little cytotoxicity to cells. The growth rate of EC9706 cells expressing NICD either transiently, or stably, was measured using the CCK- 8 method according to manufacturer's instructions. Briefly, cells were seeded into 96-well plates at about $5 \times 10^{3}$ cells per well for EC9706 cells transiently or stably expressing NICD, respectively. On the day of measuring the growth rate of EC9706 cells, $100 \mu 1$ of spent medium was replaced with an equal volume of fresh medium containing $10 \%$ CCK-8. Cells were incubated at $37^{\circ} \mathrm{C}$ for $1-4 \mathrm{~h}$, and the absorbance was finally determined at $492 \mathrm{~nm}$ using Microplate spectrophotometer.

Western blot analysis. Untreated and transfected EC9706 cells were lysed for $20 \mathrm{~min}$ in cold lysis buffer. Cell extracts were collected and centrifuged at $12,000 \mathrm{rpm}$ for $5 \mathrm{~min}$. Total proteins $(20 \mu \mathrm{g})$ from whole cell lysates were boiled for $5 \mathrm{~min}$ in $1 \mathrm{X}$ SDS buffer, resolved by $10 \%$ SDS-PAGE, and then electro-transferred to nitrocellulose membranes (Amersham, Uppsala, Sweden) by a semi-dry transferor. The membranes were blocked in $5 \%$ skimmed-milk in 
Table I. Oligonucleotide primers used for RT-PCR.

\begin{tabular}{|c|c|c|c|c|}
\hline Genes & $\begin{array}{l}\text { Size } \\
\text { (bp) }\end{array}$ & $\begin{array}{c}\text { Annealing } \\
\text { temperature } \\
\left({ }^{\circ} \mathrm{C}\right)\end{array}$ & $5^{\prime}$ primer & 3' primer \\
\hline Notch1 & 365 & 60 & 5'-GGCCACCTGGGCCGGAGCTTC-3' & 5'-GCGATCTGGGACTGCATGCTG-3' \\
\hline $\mathrm{CDK} 2$ & 415 & 58 & 5'-CCTGGACACTGAGACTGAGGGT-3' & 5'-CTCAGAATCTCCAGGGAACAGG-3' \\
\hline Cycline E & 386 & 58 & 5'-CTGGCTGAATGTTTATGTCC-3' & 5'-TCTTTGCTTGGGCTTTGTCC-3' \\
\hline Cyclin D1 & 424 & 60 & 5'-TGGAGCCCCTGAAGAAGAG-3' & 5'-AAGTGCGTTGTGCGGTAGC-3' \\
\hline P53 & 270 & 60 & 5'-TTCCTGCAGTCAGGGACAG-3' & 5'-ATACGGATTTCCTTCCACCC-3' \\
\hline $\mathrm{Bcl}-2$ & 274 & 63 & 5'-CGACTTTGCAGAGATGTCCA-3' & 5'-TAGTTCCACAAAGGCATCCC-3' \\
\hline P65 & 150 & 58 & 5'-GCGGCCAAGCTTAAGATCTGCCGAGTAAAC-3' & 5'-CGCTGCTCTAGAGAACACAATGGCCACTTGCCG-3' \\
\hline GSK3ß & 316 & 58 & 5'-CCAGTGGCGAGAAGAAAGACGAG-3' & 5'-CGGTAGTAGCGAGAACAGATGTAGGAG-3' \\
\hline B-catenin & 429 & 55 & 5'-GTTCGTGCACATCAGGATAC-3' & 5'-CGATAGCTAGGATCATCCTG-3' \\
\hline
\end{tabular}
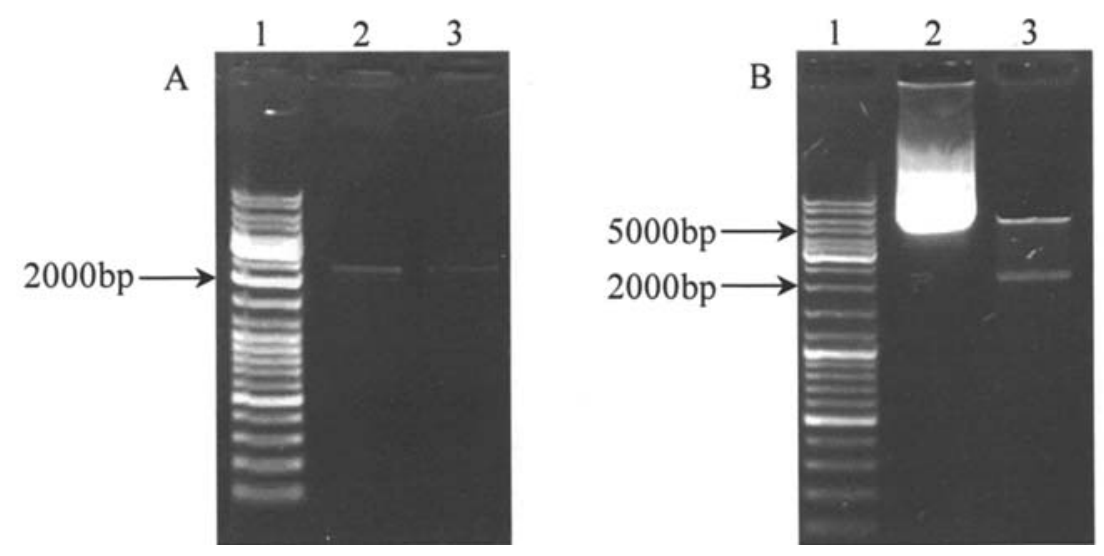

Figure 1. Construction of eukaryotic expression vector pcNICD. (A) Amplification of Notch1 cDNA fragment: lane 1, molecular size marker (Sangon GeneRuler $^{\mathrm{TM}}$ DNA Ladder Mix; Shanghai, P.R. China); lanes 2 and 3, PCR product of human Notch1 cDNA fragment. (B) Identification of eukaryotic expression vector pcNICD: lane 1, molecular size marker (Sangon GeneRuler DNA Ladder Mix); lane 2, pcNICD; lane 3, digested pcNICD with Bam $\mathrm{HI} / X b a \mathrm{I}$.

PBS-T containing $0.05 \%$ Tween-20 at RT for $2 \mathrm{~h}$, and then incubated at RT for $2 \mathrm{~h}$ with corresponding primary antibodies including Notch1, cyclin D1, cycline E, CDK2, P53, Bcl-2, P65, GSK3ß, $\beta$-catenin and $\beta$-actin (Santa Cruz) diluted in $1 \%$ skimmed-milk in PBS-T, respectively, followed by incubation with horseradish peroxidase-conjugated secondary antibodies (anti-goat, anti-rabbit or anti-mouse) (Santa Cruz). Finally, the bands of specific proteins on the membranes were developed with DAB solution according to the manufacturer's instructions. The membranes were rinsed three times with PBS-T between the incubations described above (21). Quantification of band intensity was performed using Gene Tools (UVP, Inc., Upland, USA).

Cell cycle analysis. The cells were synchronized in $\mathrm{G}_{0}$ phase by serum starvation for $24 \mathrm{~h}$ in phenol red-free RPMI with $0.1 \%$ serum. Subsequently, cells were released into complete medium containing $10 \%$ fetal bovine serum. Briefly, $1 \times 10^{6}$ of untreated and transfected EC9706 cells were harvested at 24, 48 and $72 \mathrm{~h}$ after treatment and washed in PBS, then fixed in $70 \%$ cold ethanol for $30 \mathrm{~min}$ at $4^{\circ} \mathrm{C}$. After washing in cold PBS thrice, cells were resuspended in $1 \mathrm{ml}$ of PBS solution with $40 \mu \mathrm{g}$ of propidium iodide and $100 \mu \mathrm{g}$ of RNase A for $30 \mathrm{~min}$ at $37^{\circ} \mathrm{C}$. Samples were then subjected to analysis of their DNA contents by Becton-Dickinson FACScan flow cytometer (FACScan, Becton-Dickinson, Mountain View, USA).

Apoptosis detection by flow cytometer. Untreated and transfected EC9706 cells were trypsinized at 24, 48 and $72 \mathrm{~h}$, washed with cold PBS and resuspended in PBS. Annexin V-FITC (BD Biosciences, USA) at final concentration of $1 \mu \mathrm{g} / \mathrm{ml}$ and $250 \mathrm{ng}$ of propidium iodide were added to a mixture containing $100 \mu 1$ of cell resuspension and binding buffer (BD Biosciences) each. After cells were vortexed and incubated for $15 \mathrm{~min}$ at RT in the dark, $400 \mu \mathrm{l}$ of binding buffer was added to the mixture for flow cytometric analysis.

Statistical analysis. All experiments results were from at least three independently repeated experiments. The data were carried out with one-way analysis of variance using 

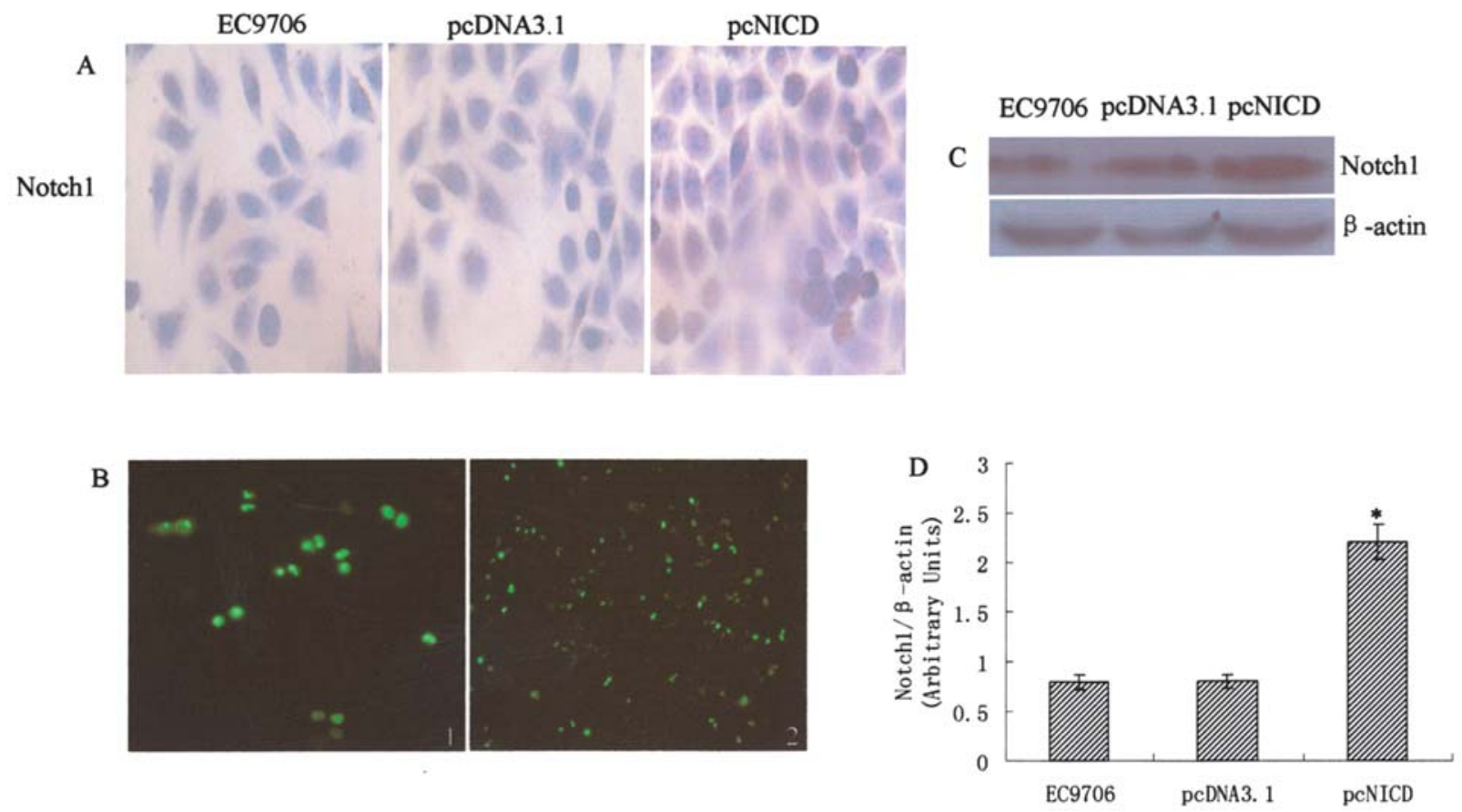

Figure 2. Expression of Notch1 protein in EC9706 cells. (A) Expression of Notch1 observed immunocytochemically in EC9706 cells transfected with and without pcDNA3.1 and pcNICD vector (magnification, x400). (B) Notch1 expressed in the cytosol and nuclei, implying an activated status (magnification, x400) (B1), and high transfection efficiency (about 95\%) in EC9706 cells transfected with pcNICD (magnification, x200) (B2). (C) Western blots analysis was performed with antibodies to Notch1 and B-actin (as a control) in EC9706 cells transfected with and without pcDNA3.1 and pcNICD vector. (D) Semiquantitive values of three independently repeated Western blots were statistically analyzed by densitometry using Gene Tools software, are expressed as means \pm SD. ${ }^{*} \mathrm{P}<0.05$, compared to those of EC9706 untreated and transfected with pcDNA3.1.
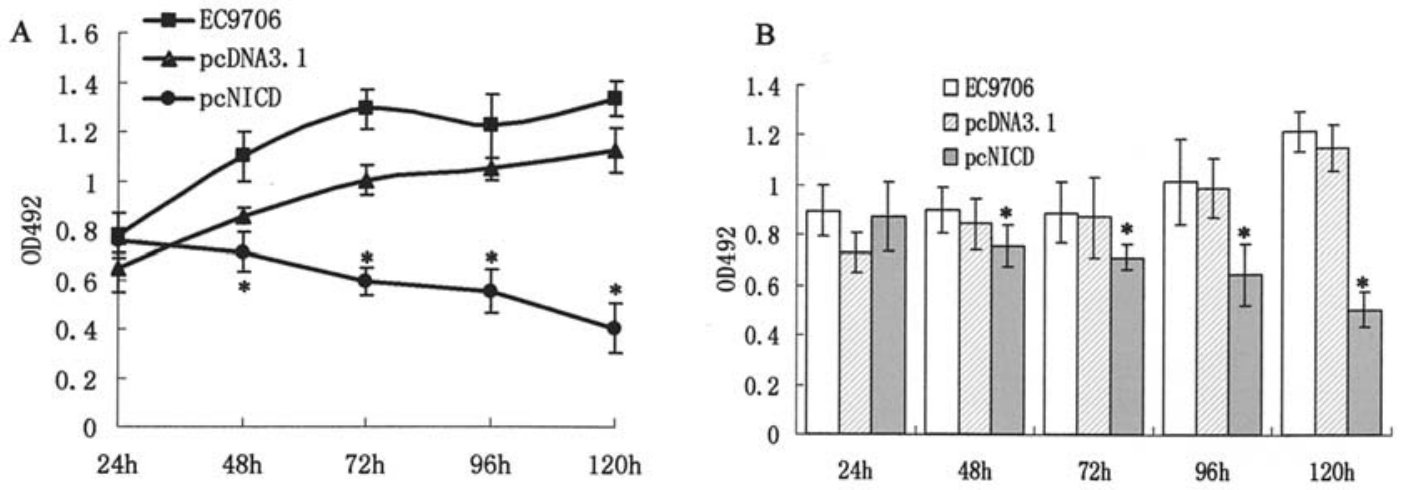

Figure 3. Activated Notch1 signaling inhibits the proliferation of human ESCC cells. (A) Growth of EC9706 cells transiently expressing Notch1 (NICD) was detected by CCK8 kit. EC9706 cells transfected with pcNICD had a decreased growth rate that was significantly different from the control EC9706 cells and EC9706 cells transfected with pcDNA3.1 ( $<<0.01)$. (B) The growth of EC9706 cells stably expressing Notch1 (NICD) was detected by CCK8 kit assay. EC9706 cells had a obvious decreased growth rate compared to the control EC9706 cells and EC9706 cells transfected with pcDNA3.1 (P<0.01). "P<0.05, compared to those of EC9706 untreated and transfected with pcDNA3.1.

SPSS version 13.0 (SPSS, Chicago, IL, USA), as described previously (19). In all statistical analyses, a P-value $<0.05$ was considered statistically significant, and all P-values were two-sided.

\section{Results}

Construction of eukaryotic expression vector pcNICD. Total RNA isolated was reverse-transcribed to cDNA first-strand, which served as a template to amplify the target gene, and a 2,058 bp of specific fragment appeared (Fig. 1A). The recovered fragments were cloned into eukaryotic expression vector pcDNA3.1 (+) to create a pcDNA3.1/Notch1 that was named pcNICD. The pcNICD was identified via double enzyme-cutting method to be the expected size (Fig. 1B) and the results of DNA sequencing were completely identical to the Notch1 sequences on GenBank.

Notch1 signaling pathway activated in EC9706 cells. To observe whether Notch1 signaling pathway exists and its activation status in EC9706 cells transfected with pcDNA3.1 and pcNICD, and untreated EC9706 cells, Notch1 protein expression levels were determined, respectively, by immunocytochemistry, immunofluorecence and Western 


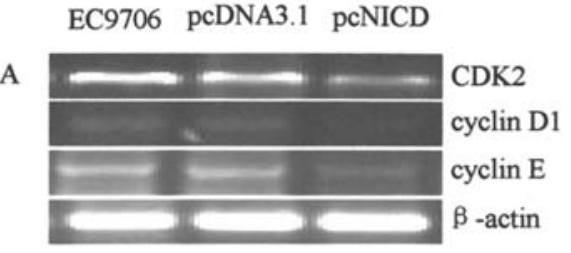

\section{EC9706 pcDNA3.1 pcNICD}

C
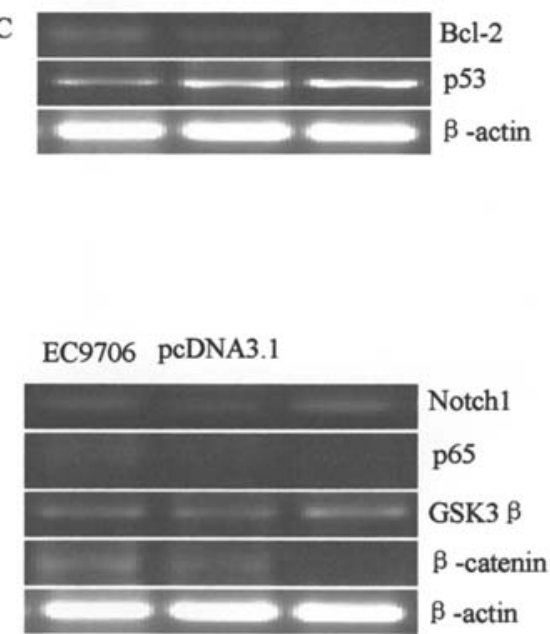
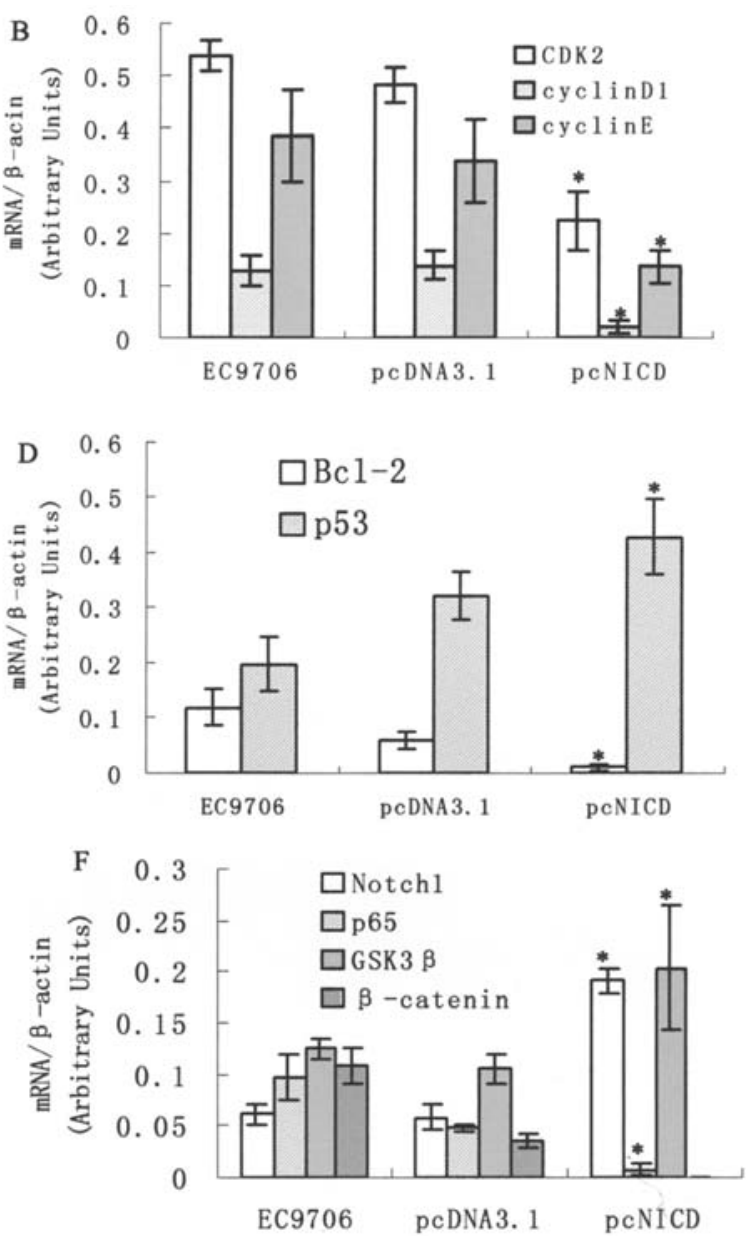

Figure 4. RT-PCR analysis of Notch1 signaling pathway-related genes. To verify the specificity of the reactions, EC9706 cells untreated and transfected with pcDNA3.1 is used as negative controls, respectively. The house-keeping gene B-actin has been used for normalization. (A, C and E) Analysis of mRNA levels of CDK2, cyclin D1, cyclin E, Bcl-2, p53, Notch1, p65, GSK3ß, B-catenin and B-actin in EC9706 cells. (B, D and F) Semi-quantitated values of mRNA levels of CDK2, cyclin D1, cyclin E, Bcl-2, p53, Notch1, p65, GSK3ß, and B-catenin to B-actin, respectively. Results from three independently repeated experiments, which were statistically analyzed by densitometry using Gene Tools software, are expressed as means \pm SD. ${ }^{*} \mathrm{P}<0.05$, compared to those of EC 9706 untreated and transfected with pcDNA3.1. Here, results of one representative experiment are reported. Levels of $\beta$-actin are shown for equal loading conditions.

blots. Expression of Notch1 protein was undetectable or weak by immunocytochemistry method in EC9706 cells untreated and transfected with pcDNA3.1, compared to cells transfected with pcNICD (Fig. 2A). Besides, expression of Notch1 protein was found both in the cytosol and nuclei of the cells transfected with pcNICD using immunofluorecence method (Fig. 2B1), suggesting that Notch1 signaling pathway is activated. Transfection efficiency was very high because of expression of Notch 1 protein in about $95 \%$ cells transfected with pcNICD (Fig. 2B2). Furthermore, the results of Western blots revealed that expression level of Notch1 protein was significantly higher $(\mathrm{P}<0.01)$ in the cells transfected with pcNICD than in the cells untreated or transfected with pcDNA3.1 (Fig. 2C and D), but there was no difference $(\mathrm{P}>0.05)$ in the expression level of Notch1 between the cells untreated and transfected with pcDNA3.1 (Fig. 2C and D). Thus, it is likely that Notch1 signaling pathway may be activated by introducing extraneous Notch1 fragment into ESCC cells.

Effect of activated Notch1 signaling pathway on proliferation of ESCC cells. The effect of activated Notch1 on proliferation of EC9706 cells were investigated using CCK-8 kit. As shown in Fig. 3A, there was no difference $(\mathrm{P}>0.05)$ in cellular proliferation rate between the cells untreated and transfected with pcDNA3.1. However, proliferation of the EC9706 cells transiently expressing NICD was inhibited significantly $(\mathrm{P}<0.01)$ compared to that of the cells untreated and transfected with pcDNA3.1. Also, proliferation of the cells stably expressing NICD was obviously inhibited $(\mathrm{P}<0.01)$ compared to that of the cells untreated and transfected with pcDNA3.1 (Fig. 3B). These findings suggest that the constitutively active Notch 1 signaling pathway, regardless of transient and stable expressions of Notch1, is able to inhibit proliferation of ESCC cells.

Identification of Notch1 signaling pathway-related genes. To investigate changes of Notch 1 signaling pathway-related genes at mRNA levels, total RNAs of untreated and transfected cells were, respectively, extracted and followed by analyzing the mRNA levels of the genes using RT-PCR. The results showed that mRNA expression of the Notch1 gene was significantly increased $(\mathrm{P}<0.01)$ in EC9706 cells transfected with pcNICD, by $\sim 3$-fold higher than that of cells untreated and transfected with pcDNA3.1 (Fig. 4E and F). Also, the mRNA expressions of the CDK2, cyclin D1 and 

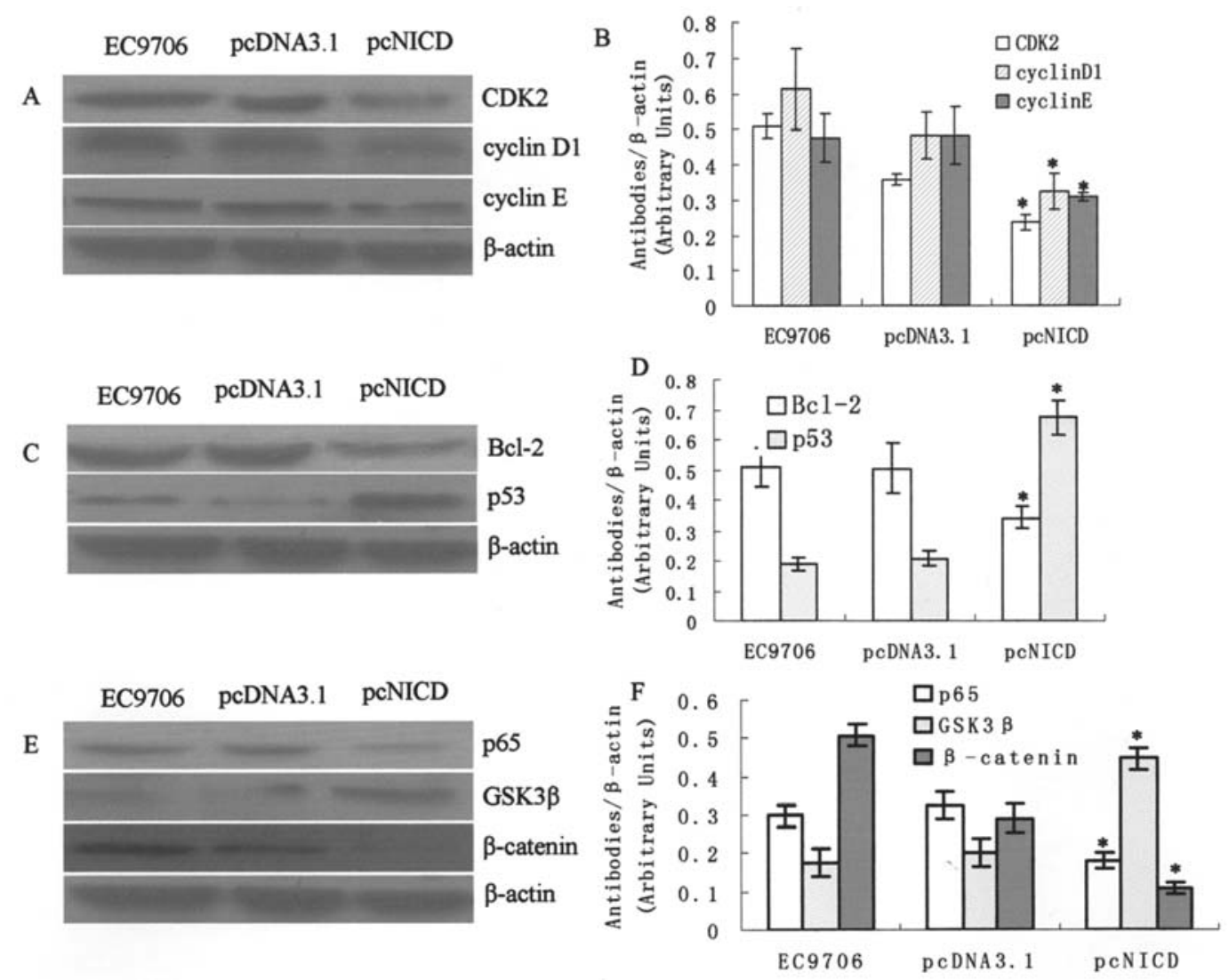

Figure 5. Western blots analysis of Notch1 signaling pathway-related genes. To verify the specificity of the reactions, EC9706 cells untreated and transfected with pcDNA3.1 is used as negative controls, respectively. The house-keeping gene B-actin has been used for normalization. (A, C and E) Analysis of proteins levels of CDK2, cyclin D1, cyclin E, Bcl-2, p53, Notch1, p65, GSK3B, B-catenin and B-actin in EC9706 cells. (B, D and F) Semi-quantitated values of proteins levels of CDK2, cyclin D1, cyclin E, Bcl-2, p53, Notch1, p65, GSK3ß, and B-catenin to B-actin, respectively. Results from three independently repeated experiments, which were statistically analyzed by densitometry using Gene Tools software, are expressed as means \pm SD. ${ }^{*} \mathrm{P}<0.05$, compared to those of EC9706 untreated and transfected with pcDNA3.1. Here, results of one representative experiment are reported. Levels of B-actin are shown for equal loading conditions.

cyclin E genes were significantly decreased in the EC9706 cells transfected with pcNICD compared to those of the cells untreated and transfected with pcDNA3.1 (Fig. 4A and B). In addition, the mRNA expressions of the Bcl-2, p53 and p65 genes involved in cell apoptosis were observed. The results demonstrated that mRNA expressions of the Bcl-2 and p65 genes were decreased in EC9706 cells transfected with pcNICD compared to those of the cells untreated and transfected with pcDNA3.1 $(\mathrm{P}<0.05)$, whereas mRNA expression of the p53 gene was increased $(\mathrm{P}<0.05)$ (Fig. 4C-F). Subsequent findings indicated that the mRNA expression of the GSK3ß gene was obviously increased $(P<0.01)$, but the mRNA expression of the $\beta$-catenin gene was significantly decreased $(\mathrm{P}<0.01)$ in the EC9706 cells transfected with pcNICD, compared to that of the cells untreated and transfected with pcDNA3.1, suggesting that the inhibitory effect of active Notch 1 signaling pathway on proliferation of EC9706 cells may be directly associated with cell cycle regulatory proteins and apoptotic proteins.

Expression levels of Notch1 signaling pathway-related proteins in EC9706 cells. According to the results of semiquantitative RT-PCR mentioned above, to determine the mechanism of proliferation reduction, we carried out Western blots analysis for various cell cycle regulatory proteins and proteins related to apoptosis and Wnt signaling pathway. As shown in Fig. 5, in the cells transfected with pcNICD, proteins levels of p65, Bcl-2, CDK2, cyclin D1 and cyclin E were obviously reduced in all three separate experiments $(\mathrm{P}<0.01)$, whereas level of p53 protein was significantly increased $(\mathrm{P}<0.01)$ compared to that of the cells untreated and transfected with pcDNA3.1. In addition, compared to that of the cells untreated and transfected with pcDNA3.1, GSK3ß, protein expression was up-regulated and B-catenin protein expression was down-regulated in the cells transfected with pcNICD, suggesting that the inhibition of active Notch1 signaling pathway on the proliferation of EC9706 cells may be mediated by Wnt signaling pathway.

Activated Notch1 signaling pathway induces cell cycle arrest and apoptosis. To characterize the mechanisms underlying the proliferation inhibition by activated Notch1 signaling pathway, we analyzed cell cycle kinetics in EC9706 cells untreated, transfected with pcDNA3.1 and pcNICD. As showed in Fig. 6A and $\mathrm{C}$, there were higher proportions of cells $(74.5 \%)(\mathrm{P}<0.05)$ in EC9706 cells transfected with pcNICD at $\mathrm{G}_{0} / \mathrm{G}_{1}$ phase than that in cells untreated (59.1\%) and transfected with pcDNA3.1 (59.0\%). The proportion of the cells transfected with pcNICD at S phase was decreased gradually from 10.3 to $7.1 \%$, indicating that the synthesis 
A
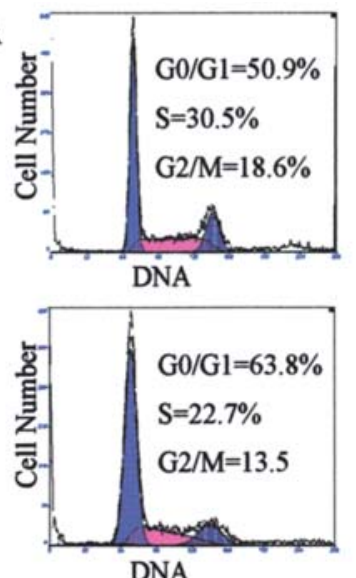

DNA

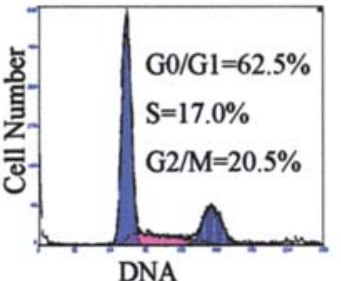

EC9706
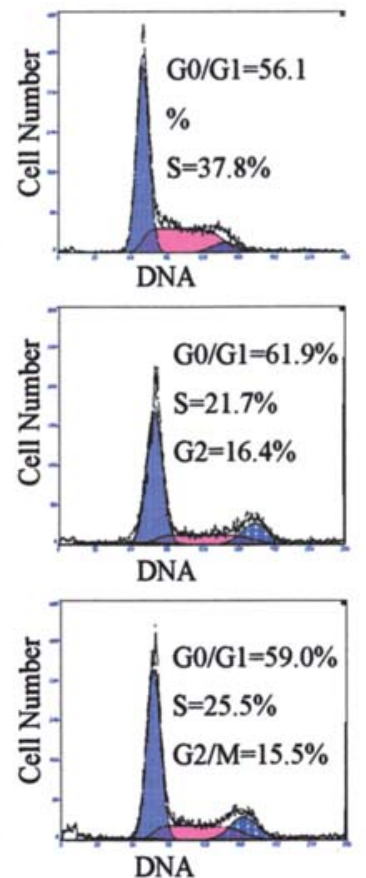

pcDNA3.1
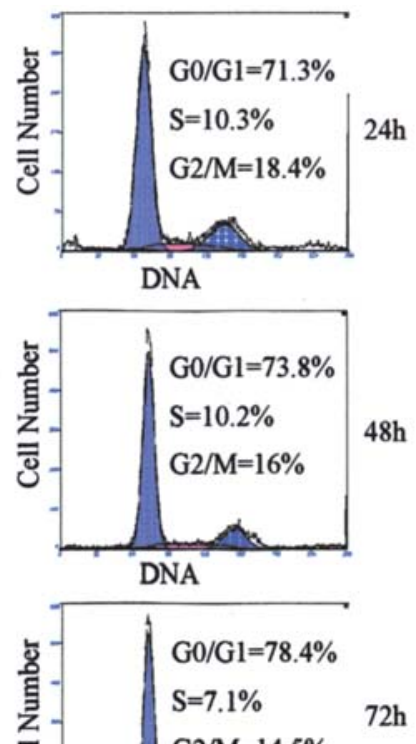

$\mathrm{G} 2 / \mathrm{M}=14.5 \%$ $72 \mathrm{~h}$

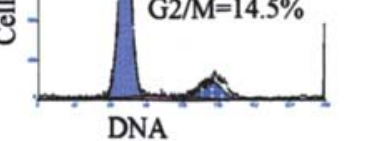

peNICD

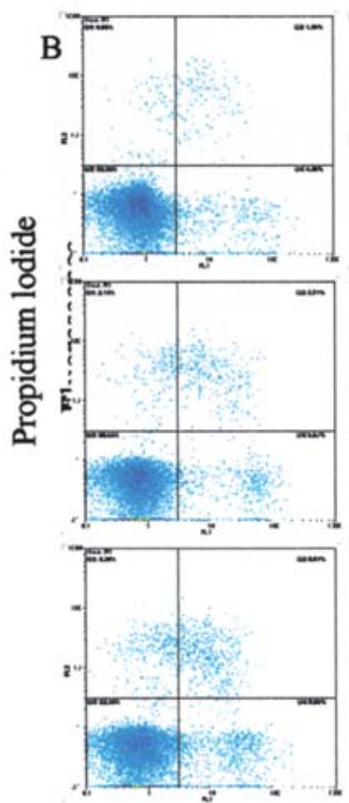

EC9076
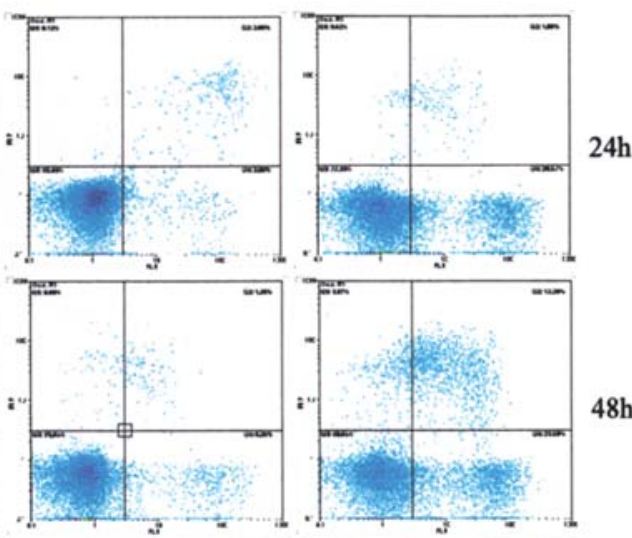

$24 \mathrm{~h}$
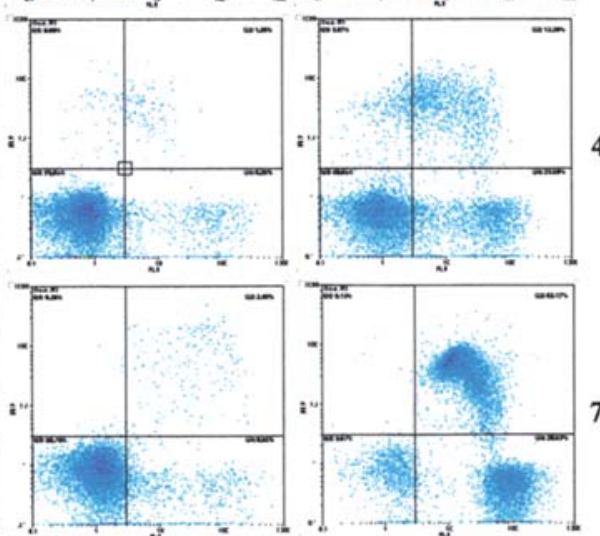

pcDNA3.1

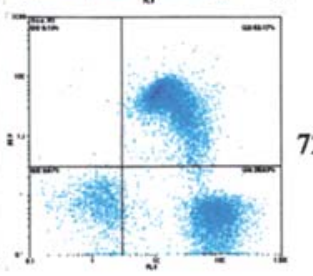

pcNICD

Annexin V-FITC
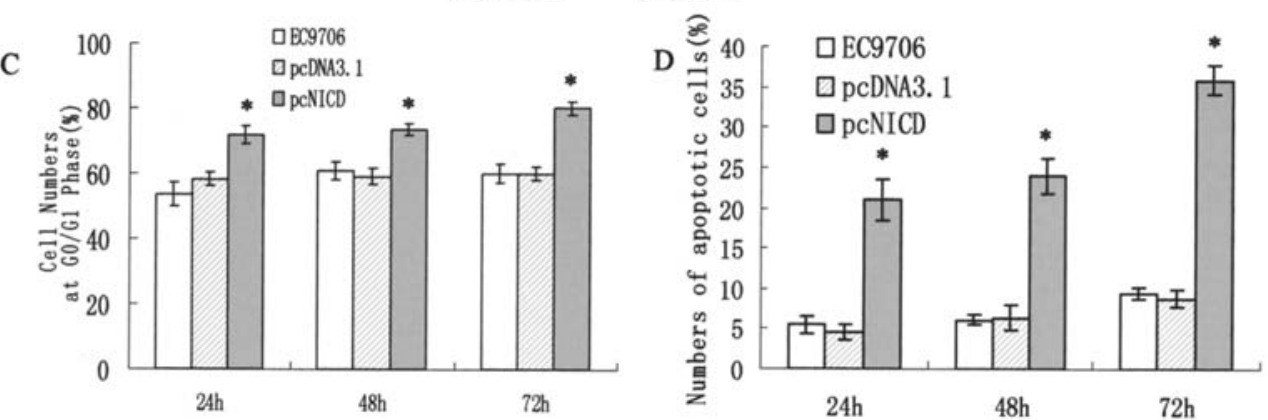

Figure 6. Activation of Notch1 signaling induces cell cycle arrest and apoptosis in EC9706 cells. EC9706 cells untreated and transfected with pcDNA3.1 and pcNICD were seeded in 6-well plates containing 10\% FBS-RPMI-1640 and harvested at 24, 48 and 72 h, respectively, followed by apoptosis assay and cell cycle analysis using the Annexin V-FITC apoptosis detection kit and propidium iodide staining, respectively. (A) Transient expression of activated Notch1 caused $\mathrm{G}_{0} / \mathrm{G}_{1}$ arrest in EC9706 cells. (B) Transient expression of activated Notch1 induced cell apoptosis in EC9706 cells. (C and D) Results derived from three independent experiments are expressed as means \pm SD. ${ }^{*} \mathrm{P}<0.05$, compared to those of EC9706 untreated and transfected with pcDNA3.1. 
of DNA was gradually prohibited due to active Notch1 signaling pathway. Cell cycle distribution analysis demonstrated that the cell increase $(P<0.05)$ at $G_{0} / G_{1}$ phase in the cells transiently expressing NICD suggests that active Notch1 signaling pathway induces G0/G1 cell cycle arrest in EC9706 cells.

Additionally, apoptotic rates of EC9706 cells untreated, transfected with pcDNA3.1 and pcNICD were analyzed by flow cytometer. The result revealed that there was no prominent difference in EC9706 cells untreated and transfected with pcDNA3.1 in the apoptotic rate of EC9706 cells (P>0.05) (Fig. 6B and D); however, the apoptosis ratio of EC9706 cells transfected with pcNICD vs. untreated and transfected with pcDNA3.1 significantly increased $(\mathrm{P}<0.01)$. Meantime, the apoptotic rate of the cells transfected with pcNICD was higher than that of the cells untreated and transfected with pcDNA3.1 at all of the time points, indicating that activated Notch1 signaling pathway could also evoke apoptosis in EC9706 cells.

\section{Discussion}

Notch1 is a multifunctional transmembrane receptor that plays an important role in cellular differentiation and development (22). Several studies have demonstrated that Notch1 signaling pathway is involved in malignant transformation and tumorigenesis (23). However, the role of Notch1 in cancer cells remains controversial. Recent studies on Notch1 signaling pathway in cancer biology help to understand that Notch1 can act either as a tumor suppressor $(17,24)$ or as an oncogene $(25)$, suggesting that the effects of Notch1 signaling pathway are cell context-specific. Overexpression of Notch1 gene causes growth inhibition of human papilloma virus-positive cervical cancinoma cells, prostate cancer and apparent reduction in small lung cancer cells (26-28). Our results revealed that active Notch1 signaling pathway could inhibit the proliferation of ESCC cells. Therefore, Notch1 may serve as a tumor suppressor in human ESCC cells, and modulation of Notch 1 signaling pathway may provide a valuable tool to manipulate the transformed state of ESCC cells.

In the present study, we have demonstrated that NICD accumulated in the cytosol and nuclei of EC9706 cells, implying that the Notch1 signaling pathway can be activated in ESCC cells by introducing extraneous Notch1 fragment. In addition, expression level of the Notch1 protein was obviously increased in the EC9706 cells transfected with pcNICD, compared to that of the cells untreated or transfected with pcDNA3.1, suggesting that Notch1 signaling pathway may be tightly associated with the development of ESCC. Therefore, it is likely that this activation of the Notch1 signaling pathway plays an important role in the development of ESCC cells.

Cyclins and CDKs play important roles in controlling major checkpoints in the cell cycle (29). Protein levels and kinase activities of cyclins A, D1 and E and CDK4 are significantly elevated in HCC (30). Recent studies have also linked cyclin D1 to cell proliferation, the activation of cyclin D1 at G1 phase are necessary for cell proliferation (31). In ESCC cells overexpressing active Notch1, we observed down-regulation of cyclin D1, cyclin E and CDK2, which were considered to be correlated with the altered cell cycle distribution phenotype and growth suppression, also, cell numbers at $\mathrm{G}_{0} / \mathrm{G}_{1}$ phase in EC9706 cells transfected with pcNICD markedly increased compared to that in EC9706 cells untreated or transfected with pcDNA3.1, suggesting the proliferation of ESCC cells was suppressed due to the expression changes of cell cycle protein, cyclin D1, cyclin E and CDK2. In human ESCC tissues, increased expression level of cyclin D1 speeded the progress of ESCC and led to poor prognosis (32). It has been reported that overexpression of cyclin E was a general phenomenon associated with oncogenesis of breast cancer (33). Coupled with the above observations together; our results demonstrate that cyclins D1 and E may play an important role in development of ESCC cells.

We forwarded the question regarding how Notch1 signaling pathway induced apoptosis in EC9706 cells by assessing the effect of active Notch1 signaling pathway on the expression of the apoptosis-related proteins p65, p53 and Bcl-2. In EC9706 cells stably expressing NICD, upregulation of p53 expression and down-regulation of p65 and Bcl-2 expression were observed. Meanwhile, the result of flow cytometer revealed that the activated Notch1 was able to induce cells apoptosis. Previous studies have demonstrated that over-expression of mutant or wild-type p53 can downregulate $\mathrm{Bcl}-2$ expression, resulting in apoptotic cell death (34). Combined with these observations, our results clearly demonstrate that active Notch1 signaling pathway-induced apoptosis may be a p53-dependent and mediated through the Bcl-2, p65 pathways.

Glycogen synthase kinase $3 \beta$ (GSK3ß) and B-catenin, which is known as a major component of Wnt signaling pathway. More recently, Notch1 has been shown to function as a tumor suppressor in mouse skin through inhibition of $\beta$-catenin signaling in Notch1-deficient mice. Besides, $\beta$-catenin has been suggested as a functional target gene for Notch1 signaling pathway that mediates the tumorsuppressive effect in murine skin carcinoma $(17,35)$. On the other hand, GSK3ß inactivates various proteins that are involved in cell proliferation and survival, such as B-catenin, cyclin D1, c-jun, c-myc, C/EBP and CREB (36). Overexpression of catalytically active GSK3ß was reported to induce apoptosis of Rat-1 and PC12 cells, whereas dominantnegative GSK3ß prevented apoptosis (37). The GSK3ß levels were markedly elevated in $\sim 50 \%$ of the tumors (38). Such an increase of GSK3ß in ESCC cells, to our knowledge, was not reported previously. To simply explore the relationship between Notch1 and Wnt signaling pathway, we further investigated the expression changes of the GSK3ß and $\beta$-catenin mRNAs and proteins, and found up-regulation of GSK3ß and down-regulation of ß-catenin in EC9706 cells stably expressing NICD, suggesting active Notch1 signaling pathway may induce cell cycle arrest by Wnt-mediated signaling pathway. However, further studies are needed to identify the mechanism that Notch1 signaling suppresses the proliferation of EC9706 cells and promotes cells apoptosis mediated by Wnt signaling pathway.

Taken altogether, our findings demonstrate that activation of Notch1 signaling pathway suppresses proliferation and 
induces apoptosis of EC9706 cells, suggesting the Notch1 signaling pathway may be used as a potential target for therapy of ESCC.

\section{Acknowledgements}

This study was supported by grants from Key Subject Program 'The Tenth Five-Year Plan' and '211 Project' (Education Ministry of China, 2002-2) and Key Research Program from Health Department of Henan Province (No. 20050009).

\section{References}

1. Pisani P, Parkin DM, Bray F and Ferlay J: Estimates of the worldwide mortality from 25 cancers in 1990. Int J Cancer 83: 18-29, 1999.

2. Montesano R, Hollstein M and Hainaut P: Genetic alterations in esophageal cancer and their relevance to etiology and pathogenesis: a review. Int J Cancer 69: 225-235, 1996.

3. Enzinger PC and Mayer RJ: Esophageal cancer. N Engl J Med 349: 2241-2252, 2003.

4. Artavanis-Tsakonas S, Matsuno K and Fortini ME: Notch signaling. Science 268: 225-232, 1995.

5. Kidd S, Lieber T and Young MW: Ligand-induced cleavage and regulation of nuclear entry of Notch in Drosophila melanogaster embryos. Genes Dev 12: 3728-3740, 1998.

6. Schroeter EH, Kisslinger JA and Kopan R: Notch-1 signalling requires ligand-induced proteolytic release of intracellular domain. Nature 393: 382-386, 1998.

7. Struhl G and Adachi A: Nuclear access and action of notch. Cell 93: 649-660, 1998.

8. Yanagawa S, Lee JS, Kakimi K, Matsuda Y, Honjo T and Ishimoto A: Identification of Notch1 as a frequent target for provirus insertional mutagenesis in T-cell lymphomas induced by leukemogenic mutants of mouse mammary tumor virus. J Virol 74: 9786-9791, 2000.

9. Osborne B and Miele L: Notch and the immune system. Immunity 11: 653-663, 1999.

10. Ohishi K, Katayama N, Shiku H, Varnum-Finney B and Bernstein ID: Notch signalling in hematopoiesis. Semin Cell Dev Biol 14: 143-150, 2003.

11. Weijzen S, Rizzo P, Braid M, et al: Activation of Notch-1 signaling maintains the neoplastic phenotype in human Rastransformed cells. Nat Med 8: 979-986, 2002.

12. Radtke F and Raj K: The role of Notch in tumorigenesis: oncogene or tumour suppressor? Nat Rev Cancer 3: 756-767, 2003.

13. Nickoloff BJ, Osborne BA and Miele L: Notch signaling as a therapeutic target in cancer: a new approach to the development of cell fate modifying agents. Oncogene 22: 6598-6608, 2003.

14. Bellavia D, Campese AF, Vacca A, Gulino A and Screpanti I: Notch3, another Notch in $\mathrm{T}$ cell development. Semin Immunol 15: 107-112, 2003.

15. Tonon G, Modi S, Wu L, et al: t(11;19)(q21;p13) translocation in mucoepidermoid carcinoma creates a novel fusion product that disrupts a Notch signaling pathway. Nat Genet 33: 208-213, 2003.

16. Enlund F, Behboudi A, Andren Y, Oberg C, Lendahl U, Mark J and Stenman G: Altered Notch signaling resulting from expression of a WAMTP1-MAML2 gene fusion in mucoepidermoid carcinomas and benign Warthin's tumors. Exp Cell Res 292: 21-28, 2004.

17. Nicolas M, Wolfer A, Raj K, et al: Notch1 functions as a tumor suppressor in mouse skin. Nat Genet 33: 416-421, 2003.

18. Tian F, Zang WD, Hou WH, Liu HT and Xue LX: Nuclear factor- $\kappa \mathrm{B}$ signaling pathway constitutively activated in esophageal squamous cell carcinoma cell lines and inhibition of growth of cells by small interfering RNA. Acta Biochim Biophys Sin 38: 318-326, 2006.
19. Hou GQ, Xue LX, Lu ZM, Fan TL, Tian F and Xue YL: An activated mTOR/p70S6K signaling pathway in esophageal squamous cell carcinoma cell lines and inhibition of the pathway by rapamycin and siRNA against mTOR. Cancer Lett (In press).

20. Lee CH, Chang SC, Chen CJ and Chang MF: The nucleolin binding activity of hepatitis delta antigen is associated with nucleolus targeting. J Biol Chem 273: 7650-7656, 1998.

21. Momota H, Nerio E and Holland EC: Perifosine inhibits multiple signaling pathways in glial progenitors and cooperates with temozolomide to arrest cell proliferation in gliomas in vivo. Cancer Res 65: 7429-7435, 2005.

22. Artavanis-Tsakonas S, Rand MD and Lake RJ: Notch signaling: cell fate control and signal integration in development. Science 284: 770-776, 1999 .

23. Maillard I, He Y and Pear WS: From the yolk sac to the spleen: new roles for Notch in regulating hematopoiesis. Immunity 18 587-589, 2003.

24. Rangarajan A, Talora C, Okuyama R, et al: Notch signaling is a direct determinant of keratinocyte growth arrest and entry into differentiation. EMBO J 20: 3427-3436, 2001.

25. Oishi K, Kamakura S, Isazawa Y, et al: Notch promotes survival of neural precursor cells via mechanisms distinct from those regulating neurogenesis. Dev Biol 276: 172-184, 2004.

26. Sriuranpong V, Borges MW, Ravi RK, Arnold DR, Nelkin BD, Baylin SB and Ball DW: Notch signaling induces cell cycle arrest in small cell lung cancer cells. Cancer Res 61: 3200-3205, 2001.

27. Shou J, Ross S, Koeppen H, De Sauvage FJ and Gao WQ: Dynamics of notch expression during murine prostate development and tumorigenesis. Cancer Res 61: 7291-7297, 2001.

28. Talora C, Sgroi DC, Crum CP and Dotto GP: Specific downmodulation of Notch1 signaling in cervical cancer cells is required for sustained HPV-E6/E7 expression and late steps of malignant transformation. Gene Dev 16: 2252-2263, 2002.

29. Sherr CJ: Cancer cell cycles. Science 274: 1672-1677, 1996.

30. Masaki T, Shiratori Y, Rengifo W, et al: Cyclins and cyclindependent kinases: comparative study of hepatocellular carcinoma vs. cirrhosis. Hepatology 37: 534-543, 2003.

31. Stacey DW: Cyclin D1 serves as a cell cycle regulatory switch in actively proliferating cells. Curr Opin Cell Biol 15: 158-163, 2003.

32. Mega S, Miyamoto M, Ebihara Y, et al: Cyclin D1, E2F1 expression levels are associated with characteristics and prognosis of esophageal squamous cell carcinoma. Dis Esophagus 18: 109-113, 2005.

33. Scott KA and Walker RA: Lack of cyclin E immunoreactivity in non-malignant breast and association with proliferation in breast cancer. Br J Cancer 76: 1288-1292, 1997.

34. Basu A and Haldar S: The relationship between Bcl2, Bax and p53: consequences for cell cycle progression and cell death. Mol Hum Reprod 4: 1099-1109, 1998.

35. Kang DE, Soriano S, Xia X, Eberhart CG, De Strooper B, Zheng $\mathrm{H}$ and Koo EH: Presenilin couples the paired phosphorylation of beta-catenin independent of axin: implications for beta-catenin activation in tumorigenesis. Cell 110: 751-762, 2002.

36. Woodgett JR: Judging a protein by more than its name: GSK-3. Sci STKE RE12, 2001.

37. Pap M and Cooper GM: Role of glycogen synthase kinase-3 in the phosphatidylinositol 3-kinase/Akt cell survival pathway. J Biol Chem 273: 19929-19932, 1998.

38. Gotoh J, Obata M, Yoshie M, Kasai S and Ogawa K: Cyclin D1 over-expression correlates with beta-catenin activation, but not with H-ras mutations, and phosphorylation of Akt, GSK3 beta and ERK $1 / 2$ in mouse hepatic carcinogenesis. Carcinogenesis 24: 435-442, 2003. 